\title{
It's Been a Long Journey: Do We Know Where We Are Going?
}

\author{
Frank P. Paloucek ${ }^{1}$ (D) $\cdot$ Michele Zell Kanter $^{2}$
}

Received: 2 April 2021 / Revised: 6 April 2021 / Accepted: 6 April 2021 / Published online: 21 April 2021

(C) American College of Medical Toxicology 2021

When we began our clinical practices in the 1980s, we both were intrigued by and drawn to clinical toxicology. In that era, the commonly encountered "difficult to manage" overdoses were aspirin, iron, theophylline, and tricyclic antidepressants. There were very few delayed release preparations, calcium channel blockers, second-generation antipsychotics, or serotonin reuptake inhibitors available.

Of the common poisonings, acetaminophen was the easiest to manage. We had a relatively new antidote and a validated nomogram. The most difficult aspect of managing the ingestion was n-acetylcysteine (NAC) administration because of both its odor if given orally and the need to compound an inhalational dosage form for intravenous administration. Patients given NAC within 24 hours of acetaminophen ingestion rarely died and the incidence of hepatoxicity was quite low [1].

The study by Baum et al. in this issue of the Journal comparing "capped" versus "uncapped" dosing strategies of Acetadote ${ }^{\circledR}$ in obese patients with an acetaminophen overdose demonstrates the challenges we continue to face managing acetaminophen poisoned patients and the issues that we tussle with [2]. Much has changed managing these patients; much is still unknown. Some of these challenges will be addressed below.

Clinical toxicology is plagued by imprecise terminologies. What is meant by the terms "obesity," "acute versus chronic ingestion," and "serial doses of activated charcoal" whether for the purpose of decontamination or elimination enhancement? Ambiguous terms lead to misinterpretation of information and errors in management, and truly demand standardization.

Supervising Editor: Mark B. Mycyk, MD

Frank P. Paloucek paloucek@uic.edu

1 Department of Pharmacy Practice, University of Illinois College of Pharmacy, 833 S. Wood Street, Chicago, IL 60612, USA

2 Toxikon Consortium, Division of Toxicology, Department of Emergency Medicine, Cook County Health, 1950 West Polk Street, 7th floor, Chicago, IL 60612, USA
Obesity, in relation to the dosing of drugs and the concept of volume of distribution, is a construct that was originally based on life insurance actuarial tables that are dynamic in nature and change over time [3]. The average weight of an adult and life expectancy has been increasing since the 1970s.

Early in the treatment of acetaminophen poisonings, there were no weight capped dosing recommendations. It was assumed that actual body weight would be used to calculate the dose of NAC in all patients. Thus, one would have anticipated that the morbidity and mortality of acetaminophen poisonings would have dropped as actual body weights increased, given that larger NAC doses were being administered. Yet this did not occur.

There is no evidence of a linear relationship between weight and liver or kidney size. Even more importantly no relationship exists between weight and function of either organ. Early efforts to determine whether a relationship existed between weight and organ function began with pharmacokinetic dosing of aminoglycosides [4]. These efforts focused on determining an adjustment or "correction" factor to the volume of distribution in the normal population. A correction factor of 0.40 was found necessary to normalize the volume of distribution in patients whose actual body weights were $\geq$ $200 \%$ of their ideal weights [5]. This correction factor became a standard dosing strategy for aminoglycosides in obese patients despite the mean value of 0.40 representing a range of 0.14-0.98 used to "fit" a normalized population value. The correction factor also became the standard for other renally eliminated drugs and eventually to hepatically metabolized drugs like acetaminophen.

Why should weight be used in calculating the toxicity risk of acetaminophen poisoning or the NAC dose? NAC is used to detoxify the toxic metabolic byproducts of acetaminophen. The original dosing was based on replacing a potential $70 \%$ deficit in glutathione stores in a $70-\mathrm{kg}$ adult with normal nutritional status (the evolution of this dosing strategy is always a must read [4]). This dosing has worked remarkably well over the years. There is no rule that a given patient would not have ingested a larger dose, i.e., a "massive" overdose, nor is there a linear relationship between weight and underlying nutritional 
status. In massive ingestions, weight-based NAC dosing in smaller than average adults or children would be inadequate while less toxic ingestions in the obese might result in adverse reactions from an excessive NAC dose.

One reason for "capping" the weight-based IV dosing method would be to minimize the risk of adverse reactions from the infusion of the first "dose," "bolus," or "bag." Yet oral administration of NAC is still an option and in fact is the least expensive option for 24-hour therapy. Prior studies examining courses of oral NAC regimens of less than 72 hours failed to achieve significance in their outcomes [6-8]. All had small study populations and used liver toxicity definitions of alanine transaminase (ALT) and aspartate aminotransferase $(\mathrm{AST}) \geq 1000 \mathrm{IU} / \mathrm{L}$.

Would results from these studies have been the same if outcomes of ALT and AST $\geq 100 \mathrm{IU} / \mathrm{L}$ were used as was done by Baum et al. in this study? Would the trials that failed to show significance when evaluating alternative durations or dosing strategies have "succeeded" if they used the "new" lower enzyme endpoints? Is this an authorization to use outcomes with more easily achievable and statistically valid results, let alone still fail to meet target sample size?

If we reconsider the appropriateness of weight-based dosing, should we not reexamine the dosing duration? Should it be the 20-hour intravenous regimen based on an assumed 4hour half-life for acetaminophen, or the original 72-hour regimen for oral administration of the inhalational dosage form to account for longer half-lives due to hepatoxicity, or other issues? [4] We are still comfortable with administering an oral NAC preparation having had the experience of using that formulation for 20 years prior to Acetadote ${ }^{\circledR}$ approval. We are familiar with its safety and the mitigation strategies that minimize the issues with patient tolerance.

So where are we going on our acetaminophen poisoning journey? Do we continue to pursue weight-based dosing, whether capped or not? Continue to use arbitrary times as endpoints? To choose endpoints based on a potential ability to meet a sample size calculation? To individualize therapy based on changes in acetaminophen concentrations? And how will the future availability of acetaminophen specific biomarkers impact our antidotal therapy? [9] Will fomepizole become part of the treatment armamentarium for acetaminophen poisoning?

These questions were unthinkable at the beginning of our careers. They have arisen over time. Many studies have since been done that have not provided much clarity and may actually have led to more confusion. There is so much to be learned. Many questions to be asked. For now, we know that "capped" NAC dosing for obese patients is not inferior to using actual weight in patients whose ALT or AST $<100$ $\mathrm{IU} / \mathrm{L}$ and it is a less expensive option for intravenous administration.

Sources of Funding None

\section{Declarations}

Conflicts of Interest None

\section{References}

1. Smilkstein MJ, Knapp GL, Kulig KW, Rumack BH. Efficacy of oral $\mathrm{N}$-acetylcysteine in the treatment of acetaminophen overdose. Analysis of the national multicenter study (1976 to 1985). N Engl J Med. 1988;319(24):1557-62. https://doi.org/10.1056/ NEJM198812153192401.

2. Baum RA, Woolum JA, Bailey AM, et al. Evaluation of dosing strategies of n-acetylcysteine for acetaminophen toxicity in patients greater than 100 kilograms: should the dosage cap be used? J Med Toxicol. 2021. https://doi.org/10.1007/s13181-021-00822-x.

3. Pai MP, Paloucek FP. The origin of the "ideal" body weight equations. Ann Pharmacother. 2000;34(9):1066-9. https://doi.org/10. 1345/aph.19381.

4. Rumack BH, Bateman DN. Acetaminophen and acetylcysteine dose and duration: past, present and future. Clin Toxicol. 2012;50(2):918. https://doi.org/10.3109/15563650.2012.659252 Erratum in: Clin Toxicol. 2021;59(4)359. doi: https://doi.org/10.1080/15563650. 2021.1885892.

5. Bauer LA, Edwards WA, Dellinger EP, Simonowitz DA. Influence of weight on aminoglycoside pharmacokinetics in normal weight and morbidly obese patients. Eur J Clin Pharmacol. 1983;24(5): 643-7. https://doi.org/10.1007/BF00542215.

6. Betten DP, Burner EE, Thomas SC, Tomaszewski C, Clark RF. A retrospective evaluation of shortened-duration oral $\mathrm{N}$-acetylcysteine for the treatment of acetaminophen poisoning. J Med Toxicol. 2009;5(4):183-90. https://doi.org/10.1007/BF03178264.

7. Betten DP, Cantrell FL, Thomas SC, Williams SR, Clark RF. A prospective evaluation of shortened course oral $\mathrm{N}$-acetylcysteine for the treatment of acute acetaminophen poisoning. Ann Emerg Med. 2007;50(3):272-9. https://doi.org/10.1016/j.annemergmed. 2006.11.010.

8. Williamson K, Wahl MS, Mycyk MB. Direct comparison of 20-hour IV, 36-hour oral, and 72-hour oral acetylcysteine for treatment of acute acetaminophen poisoning. Am J Ther. 2013;20(1):37-40. https://doi.org/10.1097/MJT.0b013e318250f829.

9. Monte AA, Sonn B, Saben J, Rumack BH, Reynolds KM, Dart RC, et al. The genomics of elevated ALT and adducts in therapeutic acetaminophen treatment: a pilot study. J Med Toxicol. 2021;17(2):160-7. https://doi.org/10.1007/s13181-020-00815-2.

Publisher's Note Springer Nature remains neutral with regard to jurisdictional claims in published maps and institutional affiliations. 